\title{
Long Noncoding RNA PRNCR1 Reduces Renal Epithelial Cell Apoptosis in Cisplatin-Induced AKI by Regulating miR-182-5p/EZH1
}

\author{
Jing Li Xing Fan Qian Wang Youlan Gong Li Guo \\ Department of Nephrology, Affiliated Hospital of Hebei University, Baoding, China
}

\author{
Keywords \\ LncPRNCR1 - miR-182-5p · EZH1 · Acute kidney injury · \\ Proliferation · Apoptosis
}

\begin{abstract}
Background/Aims: This study was designed to examine the role of long noncoding RNA PRNCR1 in cisplatin-induced acute kidney injury (AKI) in vitro and in vivo. Methods: The expression levels of PRNCR1 and miR-182-5p in cisplatin-induced AKI mice were examined. HK-2 cells were treated with cisplatin to induce cell damage. Then, the effects of PRNCR1 and miR-182-5p on cisplatin-stimulated HK-2 cell viability and apoptosis were detected by the CCK-8 and annexin VFITC/PI method. Target genes of PRNCR1 and miR-182-5p were analyzed by bioinformatics analysis and luciferase. $\boldsymbol{R} \boldsymbol{e}-$ sults: The expression level of PRNCR1 was significantly reduced in cisplatin-induced AKI mice. In addition, overexpression of PRNCR1 attenuated the damage of cisplatin to HK-2. The expression level of miR-182-5p was significantly raised in cisplatin-induced AKI mice. MiR-182-5p was negatively regulated by PRNCR1 and leaded to an upregulation of EZH1 expression. Overexpression of PRNCR1 attenuated cisplatininduced apoptosis by downregulating the miR-182-5p/
\end{abstract}

\begin{tabular}{ll}
\hline karger@karger.com & ( ) 2021 The Author(s). \\
www.karger.com/kbr & Published by S. Karger AG, Basel \\
& This article is licensed under the Creative Commons Attribution- \\
Karger & $\begin{array}{l}\text { NonCommercial-NoDerivatives 4.0 International License (CC BY- } \\
\text { NC-ND) (http://www.karger.com/Services/OpenAccessLicense). } \\
\text { Usage and distribution for commercial purposes as well as any dis- } \\
\text { tribution of modified material requires written permission. }\end{array}$
\end{tabular}

EZH1 axis. Conclusion: LncPRNCR1 reduced the apoptosis of renal epithelial cells induced by cisplatin by modulating miR182-5p/EZH1.

(c) 2021 The Author(s).

Published by S. Karger AG, Basel

\section{Introduction}

Acute kidney injury (AKI) is a common clinical syndrome that threatens global health. Its main features is the sharp decline in different degrees of renal function, including the rise of serum $\mathrm{Cr}$ and the severity of anuriafree renal failure requiring replacement therapy $[1,2]$. Due to environmental degradation and the increase of various basic diseases, the incidence of AKI is also increasing year by year [3]. Inpatients are more likely to develop AKI due to underlying diseases and iatrogenic injuries. In children, multiple organ failure, hemodynamic instability, septicemia, body fluid overload, and nephrotoxic drugs are important risk factors for AKI occurrence and mortality $[4,5]$. Modern studies have shown

Jing Li, Xing Fan, and Qian Wang contributed equally to this work.

Li Guo or Youlan Gong

Department of Nephrology, Affiliated Hospital of Hebei University

No. 212 Yuhua East Road

Baoding 071000 (China)

xq3979@163.com or xypuphqlmqlc7@163.com 
that severe AKI is closely related to the occurrence and progression of CKD, both in animal models and in humans [6]. About $15-20 \%$ of AKI patients eventually enter chronic kidneys. So far, although people have become more and more concerned about AKI, AKI is still a serious threat due to the lack of sensitive early diagnosis and effective treatment methods $[7,8]$. The important factors of patient safety in hospitals have brought a heavy medical burden to the society. Therefore, it is necessary for us to conduct in-depth research on the mechanism of AKI's occurrence and development, actively seek new targets for AKI prevention, and improve the treatment and management programs of AKI to improve patient survival and quality of life.

In recent years, more and more studies have found that long noncoding RNA can expected to become a breakthrough in disease treatment $[9,10]$. Long-chain noncoding RNA (lncRNA) is mainly located in the nucleus or cytoplasm [11, 12]. With the deepening of research, lncRNA has been shown that it has great effect in the regulation of transcriptional, posttranscriptional, and translational levels, such as RNA processing, genomic rearrangement, chromosome modification, and X-chromosome silencing [13-15]. LncRNA is widely involved in chromatin remodeling, protein modification, and other processes in vivo. It has the functions of regulating cell differentiation, cycle, metabolism, and tumor development. LncRNA is abnormally expressed in various kidney tissues and plays different roles [16]. Studies have found that IncRNA-ATB was remarkably upregulated in patients with acute rejection compared with controls. Meanwhile, lncRNA-ATB could influence the kidney cell phenotypes and impact the nephrotoxicity of immunosuppressive drug [17]. LncRNA PRNCR1 is a recently discovered novel lncRNA. LncRNA PRNCR1 is abnormally expressed in various diseases and has been shown to have gene regulation in prostate cancer; in addition, upregulation of IncRNA PRNCR1 has been noted in colorectal cancer, which promoted cell cycle and cell proliferation $[18,19]$. But the development and metastasis of lncRNA PRNCR1 in AKI remains unclear.

In recent years, the regulatory relationship of $\ln$ cRNA-microRNAs (miRNAs) has attracted the attention of more and more scholars [20]. The interactive regulation of lncRNAs and miRNAs is currently a research hotspot. miRNAs regulate cell proliferation, differentiation, growth, metabolism, and apoptosis [21]. Even small changes in the expression levels produce significant biological effects and are related to the development of many diseases [22]. In the early stage, there were a large num-

Long Noncoding RNA PRNCR1 Reduces

Renal Epithelial Cell Apoptosis ber of reports in the literature that miRNAs are associated with malignant tumors, cardiovascular diseases, and neurological diseases [23]. It has recently been found to have a key role in the progression of kidney disease [24]. It has been found that miR-21 could promote the apoptosis of renal tubular epithelial cells [25]. The expression of miR-182-5p is closely related to the development of various diseases [26]. miR-182 has been reported to be actively involved in kidney diseases. Previous research found that an in vivo experiment also validated the alleviation of the miR-182 inhibitor on I/R-induced-kidney injury and apoptosis via regulating the TCF7L2/Wnt/ $\beta$-catenin pathway [27]. The histone methylase EZH1 is a homologous protein of EZH2. The current functional studies of EZH1 focus on development and cell differentiation [28]. The main purpose of this study was to explore the mechanism of lncRNA PRNCR1 regulation of AKI.

\section{Materials and Method}

Mouse Model of Cisplatin-Induced AKI

Twenty-one clean-grade (SPF) male BALB/c mice, 6-8 weeks old, weighing about $20 \mathrm{~g}$, were purchased from the Institute of Medical Laboratory Animals, Chinese Academy of Medical Sciences. Feeding conditions were as follows: normal grade and free to eat and drink. Mice were injected intraperitoneally with cisplatin $20 \mathrm{mg} / \mathrm{kg}$, while control animals were injected with comparable amounts of saline. This study was performed in accordance with the Affiliated Hospital of Hebei University Animal Experimental Guide and approved by the Affiliated Hospital of Hebei University Hospital Animal Experimental Ethics Committee.

\section{Renal Function, Histology, and TUNEL Assay}

Mouse serum Cr was measured according to the kit (Nanjing, China), fixed with $10 \%$ formaldehyde aqueous solution, paraffinembedded, sliced, hematoxylin and eosin (HE) stained, and pathological changes of the renal tissue were observed under a light microscope. The score of tissue damage was evaluated according to the percentage of damaged tubules: " 0 " meant there was no damage, " 1 " meant the damage was $<25 \%$, " 2 " meant the damage was $25-50 \%$, " 3 " meant the damage was $50-75 \%$, and " 4 " meant the damage was $>75 \%$.

\section{Cell Culture and Treatments}

HK-2 cells were obtained from Kanglang Biotechnology Co., Ltd. (Shanghai, China). All cells were subcultured in Eagle's medium containing $10 \%$ FBS (Yilaisa, Shanghai, China). The cells were incubated at $37^{\circ} \mathrm{C}$ in a cell incubator containing $5 \% \mathrm{CO}_{2}$.

\section{Cell Transfection}

The miR-182 mimetic and anti-miR-NC were obtained from RiboBio (Guangzhou, China). The PRNCR1 cDNA sequence was amplified and introduced into a pcDNA vector (ABM, Canada) to construct a plasmid complementary DNA PRNCR1. The shRNA 
sequences targeting PRNCR1 and shNC were purchased from GenePharma Co., Ltd. (Shanghai). The cells were transiently transfected with RNAiMax and Lipofectamine 3000 with Plus Reagent (Thermo Fisher Scientific).

\section{Total RNA Extraction and Quantitative Real-Time PCR}

Total RNA from primary-cultured neurons was extracted using TRIzol reagent (Xinhaigene, Haerbin, China). qRT-PCR was performed using a ViiATM 7 real-time PCR system (Life Technologies, Grand Island, NY, USA). The expression levels of PRNCR1 and miR-182-5p were calculated by the $2^{-\Delta \Delta C T}$ method. The PRNCR1 expression level was normalized to GAPDH, while the miR-182-5p level was normalized to U6. PCR was performed using RealQ Plus $2 \times$ Master Mix Green (high Rox) (Ampliqon, Odense M, Denmark) on an Applied Biosystems StepOnePlus ${ }^{\mathrm{TM}}$ instrument. The PCR cycling conditions consisted of the first denaturation step at $95^{\circ} \mathrm{C}$ for $10 \mathrm{~min}, 40$ cycles of denaturation at $95^{\circ} \mathrm{C}$ for $15 \mathrm{~s}$, annealing at $61^{\circ} \mathrm{C}$ for PRNCR1, and then extension for $15 \mathrm{~s}$ at $72^{\circ} \mathrm{C}$. Additionally, the specificity of PCR amplicons was verified by Sanger sequencing using the Applied Biosystems 3730XL sequencer (Macrogen, Seoul, South Korea). qRT-PCR methods were performed according to reference [29]. The following primers were used:

miR-182-5p 5'-TGCGGTTTGGCAATGGTAGAAC- $3^{\prime}$

$5^{\prime}$-CCAGTGCAGGGTCCGAGGT- $3^{\prime}$

U6 5'-CTCGCTTCGGCACCACA-3'

$5^{\prime}$-AACGGTTCACGGATTTGCGT-3'

GADPH $5^{\prime}$-CCACCCAGAAGACTGTGGAT-3'

5'-TTCAGCTCAGGGATGACCTT-3'

PRNCR1, 5' CCAGATTCCAAGGGCTGATA 3',

5' GATGTTTGGAGGCATCTGGT 3’.

\section{Western Blot}

Total protein extraction was performed using RIPA solution (Thermo Fisher Scientific, Waltham, MA, USA), and protein quality was tested by the BCA assay. Protein samples $(20 \mu \mathrm{g}$ from each sample) were subjected to $12 \%$ SDS-PAGE to separate different proteins based on molecular weight, followed by transfer to PVDF membranes. Membranes were blocked by incubating with $5 \%$ skimmed milk for $1.5 \mathrm{~h}$ at room temperature, followed by incubation with rabbit anti-EZH1 (1:500), c-casp3 (1:500), Bax (1:1,000), Bcl-2 (1:1,000), and $\beta$-actin antibodies $(1: 2,000)$ (Abcam, Cambridge, MA, USA) overnight. After that, anti-rabbit secondary antibody (1:1,000, Aibixin, Shanghai, China) was added. The Western blot analysis specific-experimental method was referred to the literature [30].

\section{Bioinformatics Analysis}

The prediction of target genes of miR-182-p was performed by Starbase (http://starbase.sysu.edu.cn), TargetScan (http://www. targetscan.org/), and miRDB (mirdb.org/miRDB/) bioinformatics analysis, followed by further analysis with the KEGG system. Twenty predicted targets were identified during the first screening. Next, the secondary screening was performed by selection of genes with functional change that induced any changes in PRNCR1 and EZH1 in previous studies, which were selected as a priority over other candidates.

\section{Luciferase Reporter Gene Assay}

The PRNCR1 wild-type or mutant-binding miR-182-5p was inserted into the pMIR Basic vector (OBiO Biology, Shanghai, Chi- na) and designated as pMIR-REPOR-PRNCR1-wt or pMIR-REPOR-PRNCR1-mt. After $24 \mathrm{~h}$ of culture, cells were transfected with miR-182-5p mimic, mock control, miR-224-3p inhibitor, or inhibitor NC (GenePharma, Shanghai, China) and co-transfected with empty pMIR. After $48 \mathrm{~h}$ of transfection, luciferase activity was measured using a dual luciferase assay system (Promega).

\section{RNA Pull-Down Measurement}

The probe of miR-182-5p or PRNCR1 was biotinylated and transfected into HK2 cells. After $48 \mathrm{~h}$, the samples were nurtured in Dynabeads M-280 streptavidin (Invitrogen, Carlsbad, CA, USA). Biotinylated miR-182-5p was nurtured with magnetic beads for $10 \mathrm{~min}$.

\section{Apoptosis Analysis}

Forty-eight hours after transfection, cells were harvested by trypsinization. After centrifugation of the cells, the cells were resuspended by adding $195 \mu \mathrm{L}$ of annexin V-FITC-binding solution. After that, it was nurtured for 10-20 min, and then placed in an ice bath.

\section{CCK8 Assay}

HK-2 cells (50,000 cells per well) were seeded in 96-well plates, and $100 \mu \mathrm{L}$ of CCK8 solution (QIYBO, Shanghai, China) was added to each well at $24,48,72$, and $96 \mathrm{~h}$. After $4 \mathrm{~h}$, the absorbance at $450 \mathrm{~nm}$ was measured by a microplate reader (Bio Tek Instruments, Winooski, VT, USA).

\section{Statistical Methods}

The monitoring data were analyzed by SPSS19.0 statistical software. The results of data analysis were represented as mean \pm standard deviation (mean $\pm \mathrm{SD}$ ). Multigroup data analysis was done using one-way ANOVA. The LSD test was leveraged for subsequent analysis. $p<0.05$ indicated the difference was significant.

\section{Result}

\section{The Expression of PRNCR1 and miR-182-5p in the}

\section{Cisplatin-Induced AKI Mice and in HK-2 Cells}

First, the role of PRNCR1 and miR-182-5p in cisplatin-induced AKI was analyzed. The results are shown in Figure 1a. Serum Cr levels were significantly increased after treatment with cisplatin contrasted with the vehicle group $(p<0.05)$. As shown in Figure 1b, HE staining showed that there was no obvious change in the renal tissue. After treatment with cisplatin, the tubular epithelial cells of the mice were edematous and necrotic, and the renal interstitial had inflammatory cell infiltration. As shown in Figure 1c, renal tissue injury scores were significantly increased after treatment with cisplatin $(p<$ 0.05). As shown in Figure $1 \mathrm{~d}$ and e, the expression level of PRNCR1 in the kidney of the mice was significantly reduced $(p<0.05)$, and the expression level of miR-182- 


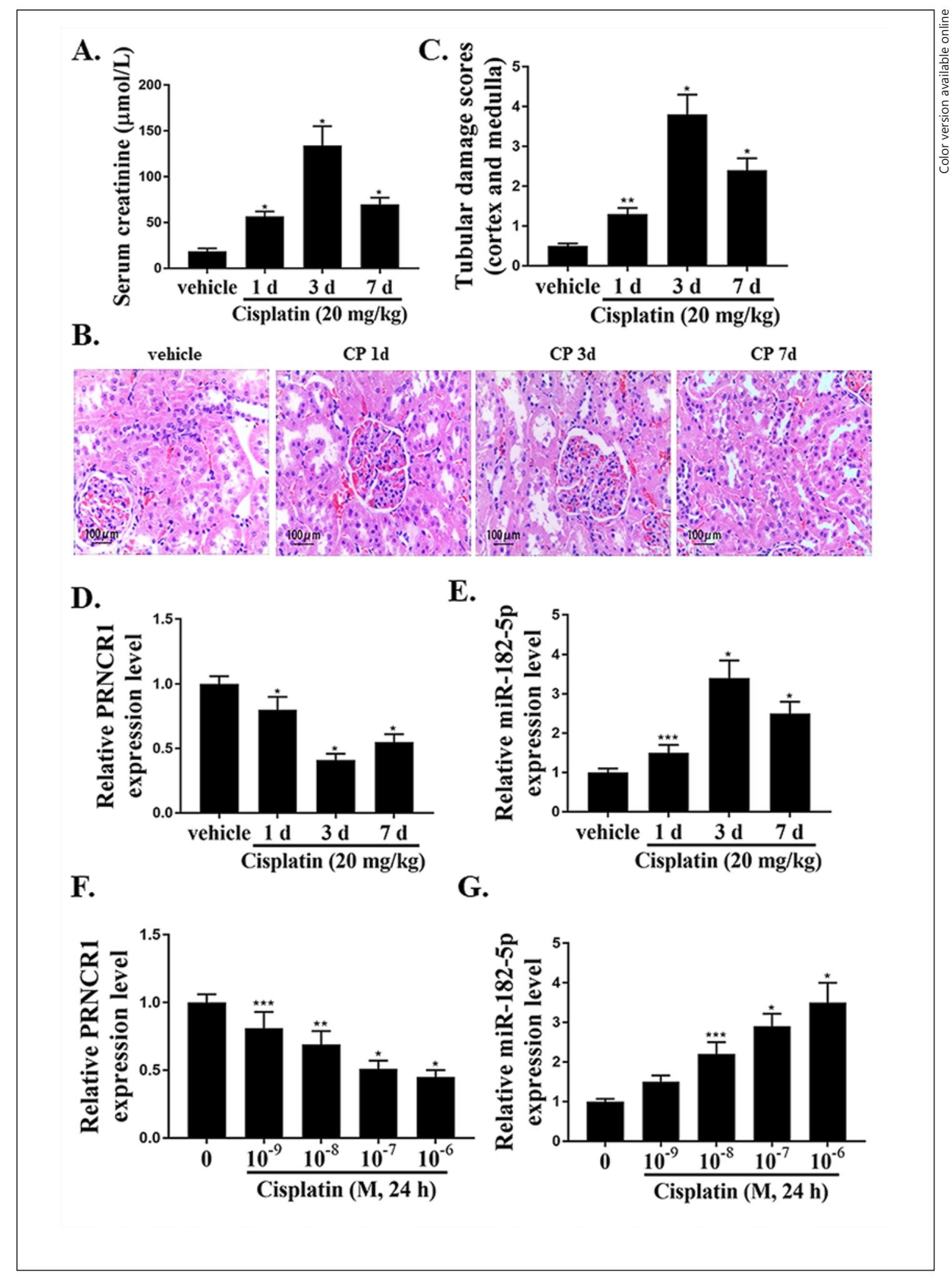

Fig. 1. Expression levels of PRNCR1 and miR-182-5p in cisplatin-induced AKI mice and HK-2 cells. A Scr level. B HE staining. C Tubular injury score. D Expression level of PRNCR1 in the kidney. $n=6$. E Expression level of miR-182-5p in the kidney. $n=6$. F PRNCR1 expression level in HK-2 cells. $n=3$. G The expression level of miR182-5p in HK-2 cells. $n=3 .{ }^{*} p<0.05,{ }^{* *} p<0.01,{ }^{* * *} p<0.001$. 


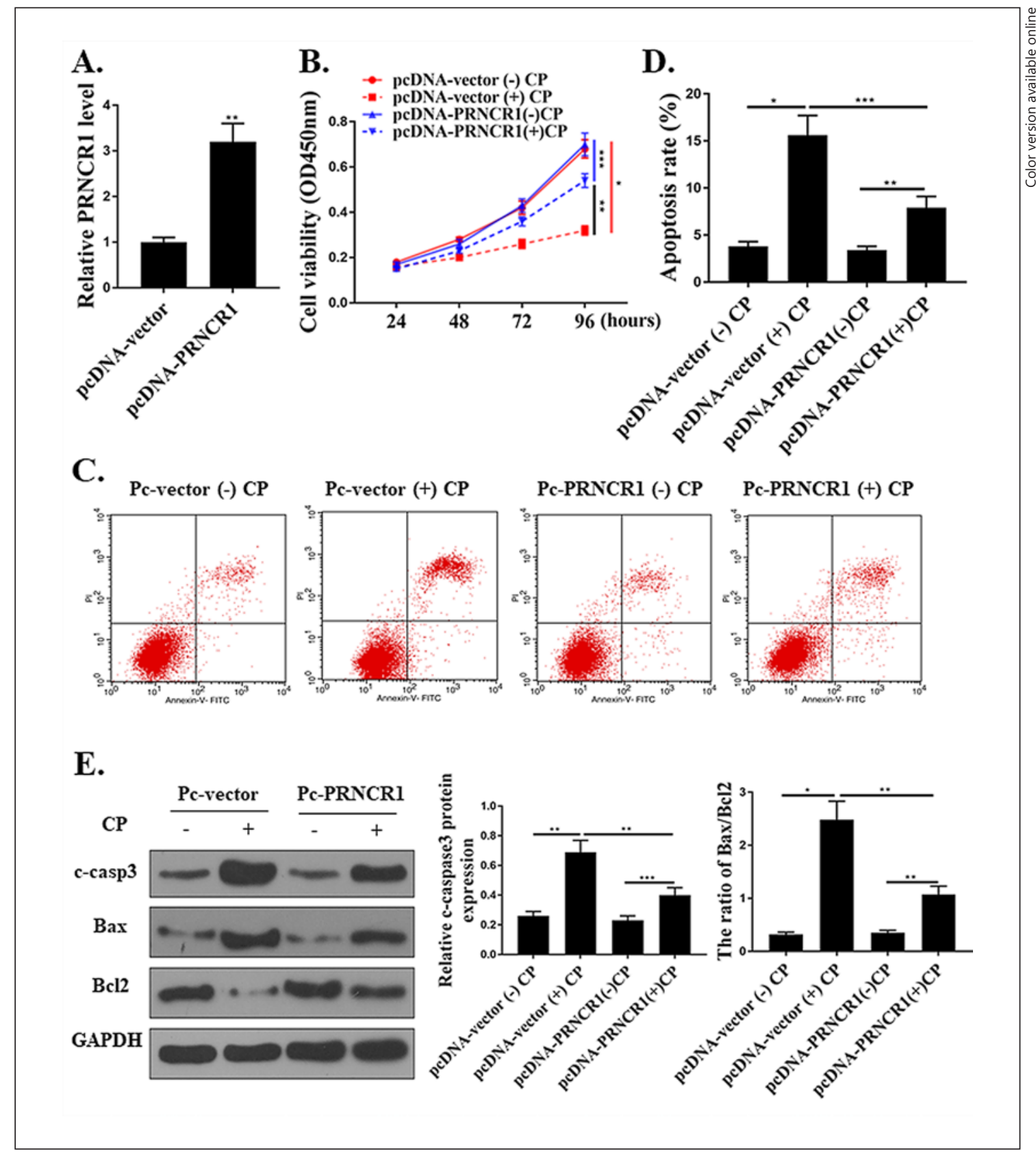

Fig. 2. The effect of PRNCR1 overexpression on cisplatin-stimulated HK-2 cell viability and apoptosis. A Expression level of PRNCR1 in HK-2 cells. B CCK8 measured cell viability. C, D Flow cytometry examined apoptosis. E Western blotting assays for caspase-3, bax and Bcl2 expression levels. CP, cisplatin. 10-6 M treatment for $24 \mathrm{~h}$. ${ }^{*} p<0.05,{ }^{* *} p<0.01,{ }^{* * *} p<0.001 . n=3$.

$5 p$ was significantly raised $(p<0.05)$. In addition, in HK-2 cells, with the increase of cisplatin concentration, the expression level of PRNCR1 was gradually decreased $(p<$ 0.05 ), and the expression level of miR-182-5p was gradually increased $(p<0.05)$ (Fig. 1f, g).
Effects of Overexpression of PRNCR1 on Cell Apoptosis in Cisplatin-Stimulated HK-2 Cells

To analyze the role of PRNCR1 in cisplatin-induced AKI, a cell model analysis was performed. The results were shown in Figure 2a. Contrasted with the pcDNA- 


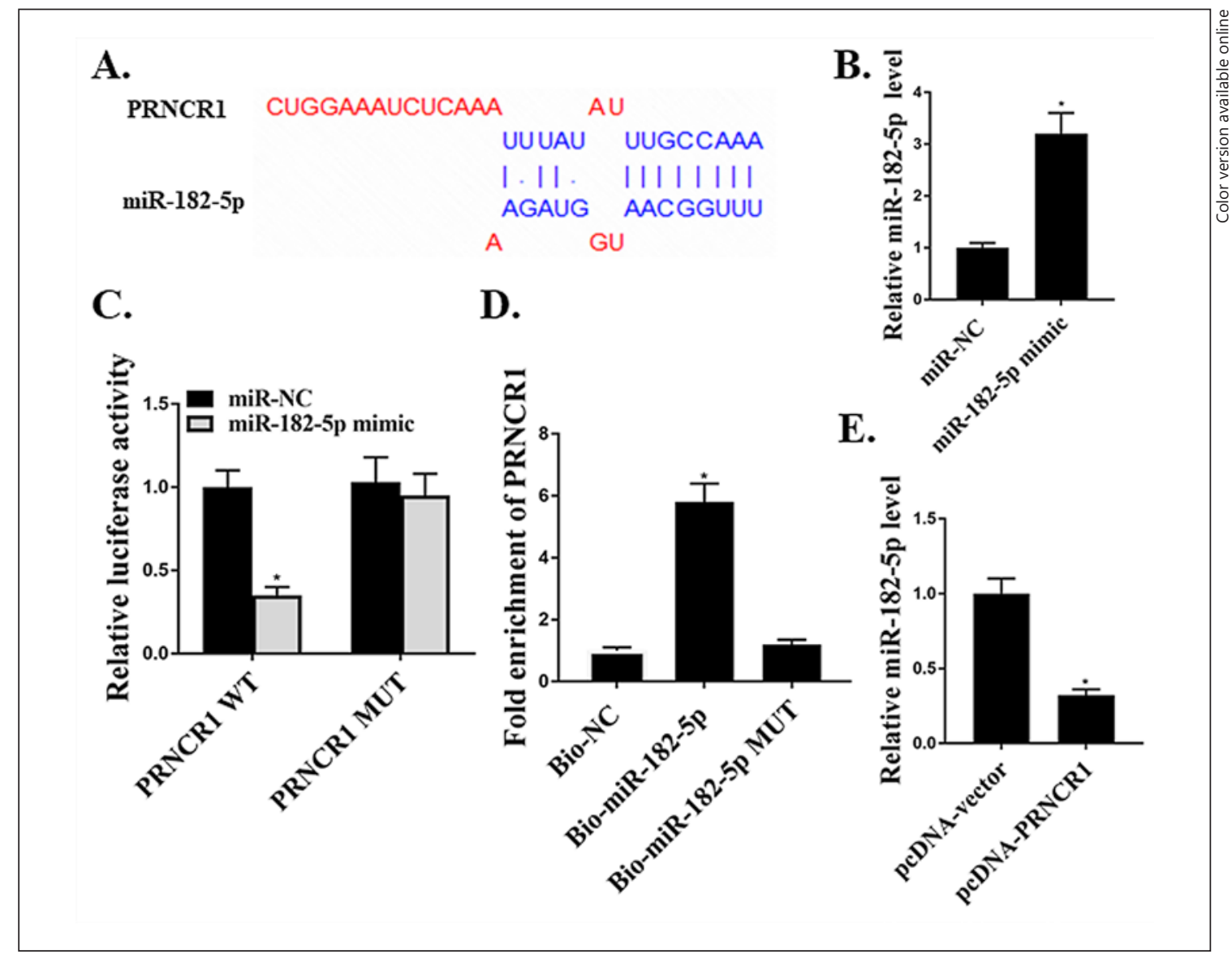

Fig. 3. Relationship between miR-182-5p and PRNCR1 in HK-2 cells. A Putative target sequence of miR-182-5p on the 3'-UTR of PRNCR1. B PRNCR1 expression levels in HK-2 cells. C Detection of luciferase activity by luciferase reporter assay. D RNA pulldown assay. E miR-182-5p expression level in HK-2 cells. ${ }^{*} p<0.05,{ }^{* *} p<$ $0.01,{ }^{* * *} p<0.001 . n=3$.

vector group, the expression level of PRNCR1 in the pcDNA-PRNCR1 group was significantly increased, indicating successful transfection $(p<0.05)$. As shown in Figure $2 \mathrm{~b}$, cisplatin treatment significantly reduced cell viability in the pcDNA-vector group $(p<0.05)$; however, PRNCR1 overexpression significantly increased cell viability after cisplatin treatment $(p<0.05)$. As shown in Figure $2 \mathrm{c}$ and $\mathrm{d}$, cisplatin treatment significantly induced apoptosis in the pcDNA-vector group $(p<0.05)$; however, PRNCR1 overexpression significantly inhibited apoptosis after cisplatin treatment $(p<0.05)$. In addition, as shown in Figure 2e, cisplatin treatment significantly upregulated the protein expression levels of c-casp3 and $\mathrm{Bax}$ in the pcDNA-vector group and downregulated Bcl$2(p<0.05)$. However, PRNCR1 overexpression significantly reduced the protein expression level of c-casp3 and Bax in cisplatin-induced cells and raised Bcl-2 $(p<0.05)$.
The original Western blot images in Figure 2 are shown in online suppl. Figure 1 (for all online suppl. material, see www.karger.com/doi/10.1159/000510157).

\section{Reciprocal Inhibition between miR-182-5p and PRNCR1 in Human Kidney Cells}

As shown in Figure 3a, bioinformatic analysis indicated that miR-182-5p was predicted to be a target gene for PRNCR1. As shown in Figure 3b, contrasted with the miR-NC group, the expression level of miR-182-5p was significantly raised in the miR-182-5p-mimic group $(p<$ $0.05)$. Luciferase activity was significantly decreased in cells transfected with miR-182-5p mimic and PRNCR1WT $(p<0.05)$, but the luciferase activity of PRNCR1MUT was not changed significantly (Fig. 3c). Furthermore, as shown in Figure 3d, PRNCR1 was pulled-down by biotinylated miR-182-5p $(p<0.05)$, but not by bioti- 
A.

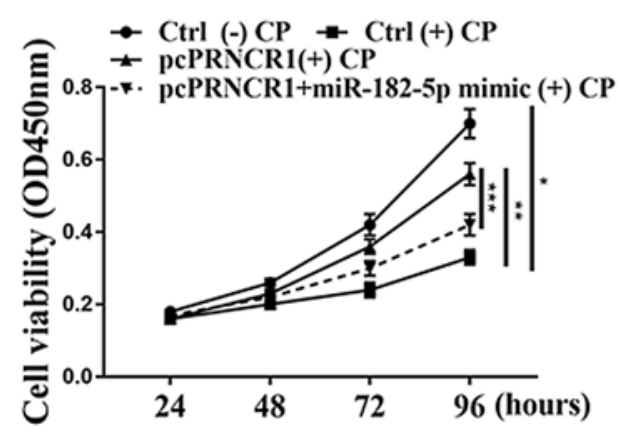

B.
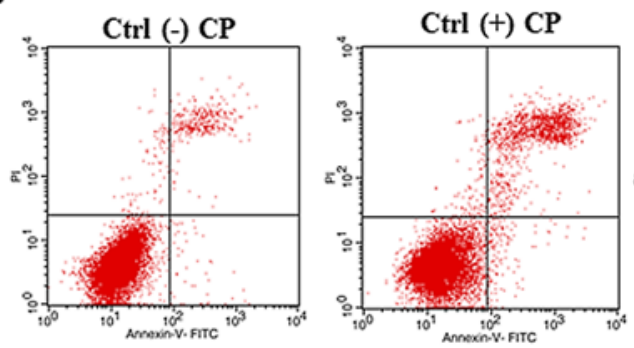

C.

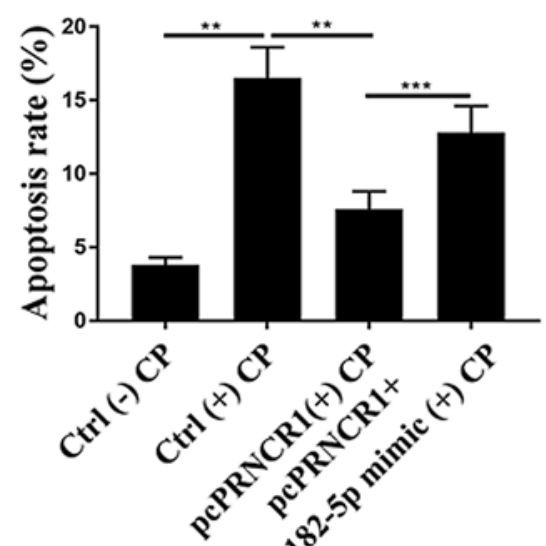

Pc-PRNCRl (+) CP+

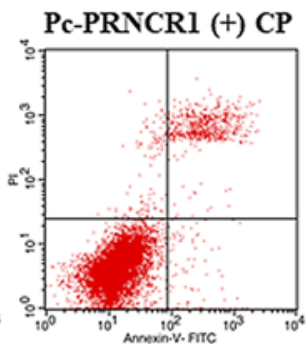

miR-182-5p mimic

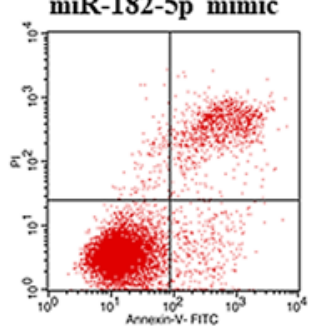

D.
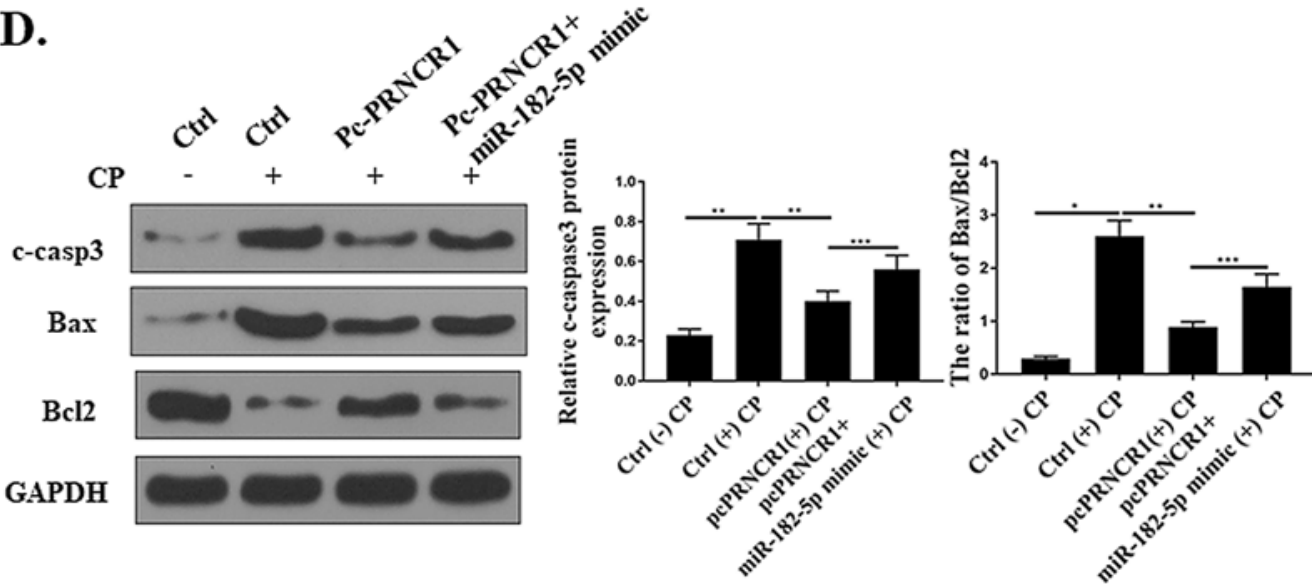

Fig. 4. Upregulation of miR-182-5p reversed PRNCR1-induced HK-2 cell apoptosis. A CCK-8 measured cell viability. B, C Flow cytometry examined apoptosis. D Protein expression levels of c-caspase-3, bax and Bcl-2. CP, cisplatin. 10-6 M treatment for 24 h. ${ }^{*} p<0.05,{ }^{* *} p<0.01,{ }^{* * *} p<0.001 . n=3$.

nylated miR-182-5p-mut. In addition, contrasted with the pcDNA-vector group, the expression level of miR$182-5 p$ was significantly reduced in the pcDNA-PRNCR1 group $(p<0.05)$ (Fig. 3e). These results indicated that PRNCR1 was capable of targeting miR-182-5p.
Upregulation of miR-182-5p Mostly Reversed PRNCR1-Induced Apoptosis in HK-2 Cells

Next, PRNCR1-induced apoptosis through miR-182$5 p$ was analyzed. As shown in Figure $4 a-c, p c P R N C R 1$ significantly raised the cell viability of cisplatin-treated 


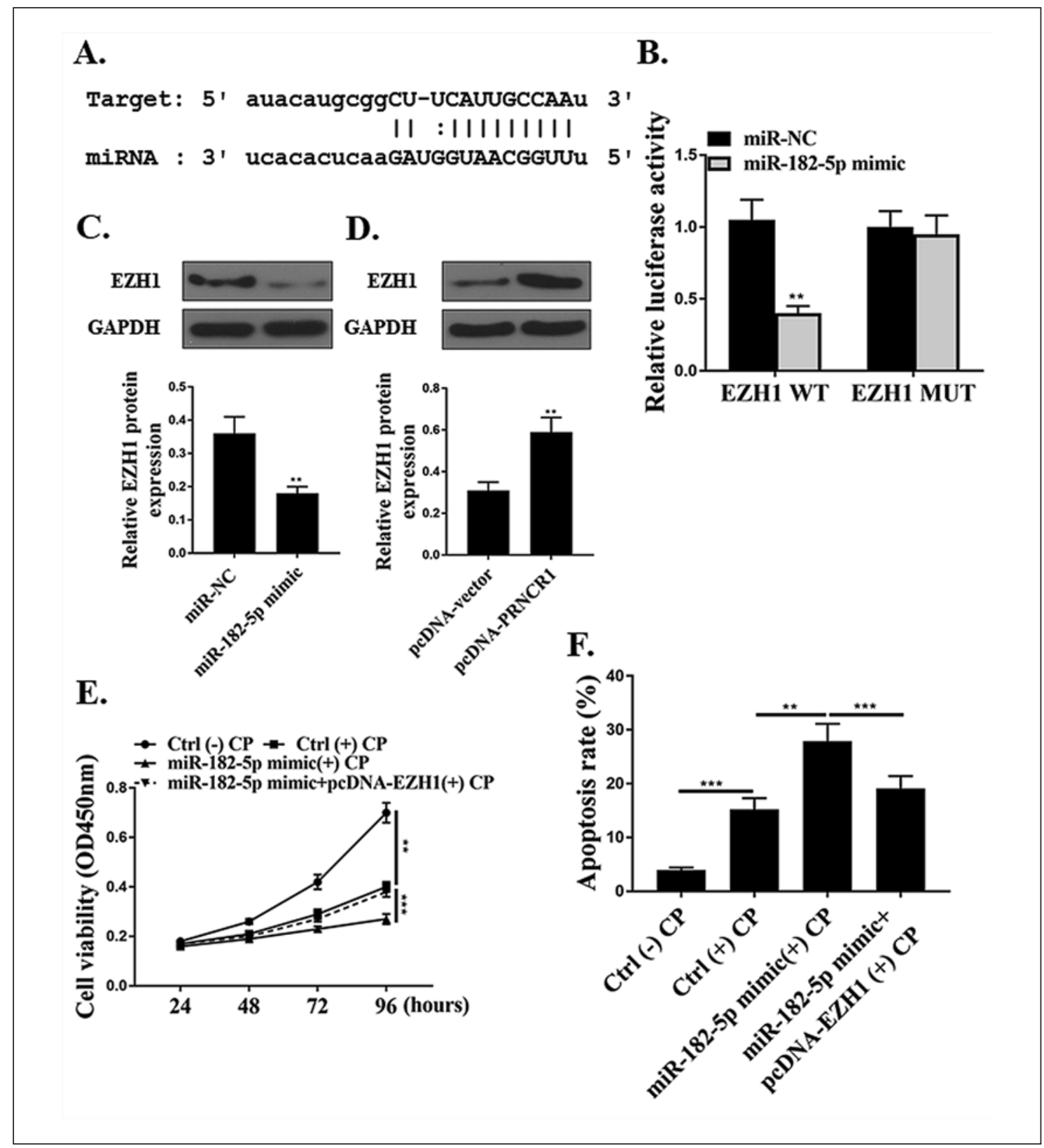

Fig. 5. The relationship between miR-182-5p and EZH1 in HK-2 cells. A Putative target sequence of miR-182-5p on the 3'-UTR of EZH1. B Detection of luciferase activity by luciferase reporter assay. C, D EZH1 protein levels in HK-2 cells. E CCK8 measured cell viability. F Flow cytometry to examine apoptosis. ${ }^{*} p<0.05,{ }^{* *} p<0.01$, *** $p<0.001 . n=3$.

HK-2 cells $(p<0.05)$ and inhibited apoptosis in cisplatintreated HK-2 cell $(p<0.05)$. Co-transfection of pcPRNCR1 with miR-182-5p mimic reversed the effect of pcPRNCR1 on cell viability and apoptosis $(p<0.05)$. As shown in Figure 4d, pcPRNCR1 significantly downregulated the expression levels of $c$-casp3 and $\operatorname{Bax}(p<0.05)$

Long Noncoding RNA PRNCR1 Reduces Renal Epithelial Cell Apoptosis and upregulated the expression level of Bcl-2 $(p<0.05)$. Co-transfection of pcPRNCR1 with miR-182-5p mimic reversed the effect of pcPRNCR1 on the expression levels of Bcl-2, c-casp3, and $\mathrm{Bax}(p<0.05)$. The original Western blot images in Figure 4 are shown in online suppl. Figure 2. 
Reciprocal Inhibition between miR-182-5p and EZH1 in Human Kidney Cells

As shown in Figure 5a, bioinformatic analysis indicated that EZH1 was predicted to be a target gene for miR-182$5 p$. Luciferase activity was significantly decreased in cells transfected with miR-182-5p mimic and EZH1-WT $(p<$ $0.05)$, but the luciferase activity of EZH1-MUT was not changed significantly (Fig. 5b). These results indicated that EZH1 was a specific target gene of miR-182-5p. Furthermore, as shown in Figure $5 \mathrm{c}$ and d, miR-182-5p mimic significantly reduced EZH1 protein expression $(p<0.05)$, while pcDNA-PRNCR1 significantly increased EZH1 protein expression $(p<0.05)$. The original Western blot images in Figure $5 \mathrm{c}$ and $5 \mathrm{~d}$ are shown in online suppl. Figures 3 and 4, respectively. Furthermore, as shown in Figure 5e and $\mathrm{f}, \mathrm{miR}-182-5 \mathrm{p}$ mimic significantly inhibited the cell viability $(p<0.05)$ and induced apoptosis $(p<0.05)$. Cotransfection of pcDNA-EZH1 with miR-182-5p mimic reversed the effect of miR-182-5p mimic on cell viability and apoptosis of cisplatin-treated HK-2 cells $(p<0.05)$.

\section{Discussion}

AKI is an important cause of secondary CKD, renal failure, and death in hospitalized patients. According to statistics, $70 \%$ of hospitalized critically ill patients have AKI, which induces a mortality rate of more than 6 times $[1,31]$. Kidney injury still lacks effective treatment methods. Therefore, the investigation of its pathogenesis and treatment methods is one of the hotspots in recent years. The causes of AKI include ischemia, drug toxicity, sepsis, and urinary tract obstruction [32]. Therefore, the study of AKI, especially renal toxic AKI, is conducive to a deeper understanding of the mechanism of AKI occurrence and repair, as well as providing new ideas and theoretical basis for the treatment of AKI.

It is well known that cisplatin is one of the classic broadspectrum chemotherapy drugs used to treat tumors but can cause severe nephrotoxicity during treatment [33]. The pathological process of renal damage caused by cisplatin mainly includes inflammation of renal tubular epithelial cells, apoptosis, and even necrosis. However, the specific pathogenesis needs further exploration [34]. This study found that SCr was significantly elevated in the model group, and renal histopathology was changed significantly, indicating successful modeling.

Recent studies have shown that some kidney diseases are also inseparable from lncRNAs $[35,36]$. With the gradual deepening of lncRNA research, many $\operatorname{lncRNAs}$ have been found to play a key role in nephropathy [37]. For example, the level of lncRNA-ATB in plasma and renal biopsy samples of the AKI group was higher, suggesting that lncRNAs can be used as biomarkers of AKI [17]. LncRNA PRNCR1 is an upcoming lncRNA that has received increasing attention. Previous studies have found that PRNCR1 plays a part in the tumors such as gastric cancer and liver cancer [38]. This study found that the expression level of PRNCR1 was reduced in mouse kidney and HK-2 cells after cisplatin treatment. Overexpression of PRNCR1 significantly increased cell viability after cisplatin treatment and inhibited apoptosis after cisplatin treatment $(p<0.05)$. The protein expression level of $c-$ casp 3 and Bax in the cells induced by cisplatin was reduced and Bcl-2 was raised. Therefore, the purpose of controlling the development of AKI can be achieved by upregulating the expression of lncRNA PRNCR1.

miRNA is very potent and is involved in almost all known biological regulatory processes, such as cell differentiation and apoptosis [39]. It can be highly expressed as a biological marker in the human circulating blood system [40]. Recent studies have shown that circulating miRNA undergo significant changes in AKI, liver damage, and heart injury [41]. Studies have found that circulating miR210 is higher in patients with clinically critical AKI than in other patients. Another study has found that plasma and urine miR-21 levels were elevated in patients with AKI after cardiac surgery and were associated with progression of AKI [42]. MiR-182-5p is a recently discovered miRNA, and it is found that miR-182-5p is abnormally expressed in various diseases [43]. There have been several reports about miR-182-5p's functions in the progression of breast cancer and lung cancer. This study found that the expression level of miR-182-5p was raised in mouse kidney and HK-2 cells after cisplatin treatment. miR-182-5p was the target gene of PRNCR1. miR-182-5p mimic significantly inhibited the cell viability and induced apoptosis. Co-transfection of pcPRNCR1 with miR-182$5 \mathrm{p}$ mimic reversed the effects of pcPRNCR1 on cell viability, apoptosis, and related apoptotic proteins. These results indicated that lncRNA PRNCR1 may inhibit the development of AKI by modulating miR-182-5p.

Recent studies have shown that interactions between miRNA and IncRNA target and regulate related pathways [44]. EZH1 is a member of the PcG protein family. PcG family plays an important role in maintaining the physiological state of cells [45]. This study found that EZH1 was a potential target of miR-182-5p, miR-182-5p mimic significantly reduced the expression level of EZH1 protein, while pcDNA-PRNCR1 significantly raised the ex- 
pression level of EZH1 protein. Co-transfection of pcDNA-EZH1 with miR-182-5p mimic reversed the effect of miR-182-5p mimic on cell viability and apoptosis of cisplatin-treated HK-2 cells. It was shown that lncRNA PRNCR1 can inhibit the development of AKI by regulating the miR-182-5p/EZH1 axis.

\section{Conclusion}

LncRNA PRNCR1 was able to inhibit the development of AKI by modulating the miR-182-5p/EZH1 axis. It suggested that lncRNA PRNCR1 may be a potential therapeutic gene for AKI, providing experimental evidence for the clinical prognosis of AKI.

\section{Statement of Ethics}

This study was performed in accordance with the Affiliated Hospital of Hebei University Animal Experimental Guide and approved by the Affiliated Hospital of Hebei University Hospital Animal Experimental Ethics Committee.

\section{Conflict of Interest Statement}

The authors declare that they have no competing interests.

\section{Funding Sources}

Not applicable.

\section{Author Contributions}

Jing Li, Xing Fan, Qian Wang, Youlan Gong, and Li Guo contributed to data analysis, drafting or revising the article, gave final approval of the version to be published, and agreed to be accountable for all aspects of the work.

\section{Availability of Data and Material}

The analyzed data sets generated during the study are available from the corresponding author on reasonable request.

\section{References}

1 Basile DP, Anderson MD, Sutton TA. Pathophysiology of acute kidney injury. Compr Physiol. 2012;2(2):1303-53.

2 O’Riordan A, Brummell Z, Sizer E, Auzinger G, Heaton N, O'Grady JG, et al. Acute kidney injury in patients admitted to a liver intensive therapy unit with paracetamol-induced hepatotoxicity. Nephrol Dial Transplant. 2011; 26(11):3501-8

3 Forni LG, Darmon M, Ostermann M, Oudemans-van Straaten HM, Pettilä V, Prowle JR, et al. Renal recovery after acute kidney injury. Intensive Care Med. 2017;43(6):855-66.

4 Göcze I, Jauch D, Götz M, Kennedy P, Jung B, Zeman F, et al. Biomarker-guided intervention to prevent acute kidney injury after major surgery: the prospective randomized bigpak study. Ann Surg. 2018;267(6):1013-20.

5 Zhang W, Yuan W, Xu N, Li J, Chang W. Icariin improves acute kidney injury and proteinuria in a rat model of pregnancy-induced hypertension. Mol Med Rep. 2017;16(5):7398404.

6 Kamar C, Ali A, Altun D, Orhun G, Sabanc1 A, Sencer A, et al. Evaluation of risk factors and development of acute kidney injury in aneurysmal subarachnoid hemorrhage, head injury, and severe sepsis/septic shock patients during icu treatment. Ulus Travma Acil Cerrahi Derg. 2017;23(1):39-45.

7 Zhou CC, Yao WT, Ge YZ, Xu LW, Wu R, Gao XF, et al. Remote ischemic conditioning for the prevention of contrast-induced acute kidney injury in patients undergoing intravascular contrast administration: a metaanalysis and trial sequential analysis of 16 randomized controlled trials. Oncotarget. 2017; 8(45):79323-36.

8 Yao Y, Hu X, Feng X, Zhao Y, Song M, Wang $\mathrm{C}$, et al. Dexmedetomidine alleviates lipopolysaccharide-induced acute kidney injury by inhibiting the NLRP3 inflammasome activation via regulating the TLR4/NOX4/NF- $\kappa \mathrm{B}$ pathway. J Cell Biochem. 2019;120(10): 18509-23.

9 Zhang J, Gao Y. Long non-coding RNA MEG3 inhibits cervical cancer cell growth by promoting degradation of P-STAT3 protein via ubiquitination. Cancer Cell Int. 2019;19: 175.

10 Bo Z, Tao W, Sicong M, Xingxing Q, Xiaoyin $\mathrm{T}$, Dan $\mathrm{C}$, et al. Long noncoding RNA ZNFX1-AS1 suppresses growth of hepatocellular carcinoma cells by regulating the methylation of miR-9. Onco Targets Ther. 2016;9:500514.

11 Dan D, Zhongyi M, Chenghai Z, Mingli S. ZFAS1: a novel tumor-related long non-coding RNA. Cancer Cell Int. 2018;18:125.

12 Liu Z, Yan Y, Cao S, Chen Y. Long non-coding RNA SNHG14 contributes to gastric cancer development through targeting miR-145/ SOX9 axis. J Cell Biochem. 2018;119(8): 6905-13.

13 Yang C, Wu K, Wang S, Wei G. Long noncoding RNA XIST promotes osteosarcoma progression by targeting YAP via miR-1955p. J Cell Biochem. 2018;119(7):5646-56.

14 Yan L, Wenwen Z, Pengying L, Yumei X, Lin $\mathrm{T}$, Weiwei $\mathrm{C}$, et al. Long non-coding rna fendrr inhibits cell proliferation and is associated with good prognosis in breast cancer. Onco Targets Ther. 2018;11:1403-12.

15 Marchese FP, Huarte M. A long noncoding rna in DNA replication and chromosome dynamics. Cell Cycle. 2017;16(2):151-2.

16 Chen Y, Qiu J, Chen B, Lin Y, Chen Y, Xie G, et al. Long non-coding RNA NEAT1 plays an important role in sepsis-induced acute kidney injury by targeting miR-204 and modulating the NF- $\kappa$ B pathway. Int Immunopharmacol. 2018;59:252-60.

17 Qiu J, Chen Y, Huang G, Zhang Z, Chen L, Na $N$. The TGF- $\beta$ activated long non-coding RNA ATB plays an important role in acute rejection of renal allografts and may impacts the postoperative pharmaceutical immunosuppression therapy. Nephrology. 2017; 22(10);769-803.

18 Gong Z-M, Tang Z-Y, Sun X-L. LNCRNA PRNCR1 regulates osteogenic differentiation in osteolysis after hip replacement by targeting miR-211-5p. Gene. 2018;673:251-61.

19 Sattarifard H, Hashemi M, Hassanzarei S, Narouie B, Bahari G. Association between genetic polymorphisms of long non?Coding rna PRNCR1 and prostate cancer risk in a sample of the iranian population. Mol Clin Oncol. 2017;7(6):1152-8. 
20 Li N, Ponnusamy M, Li M-P, Wang K, Li P-F. The role of MicroRNA and LncRNA-MicroRNA interactions in regulating ischemic heart disease. J Cardiovasc Pharmacol Ther. 2017;22(2):105-11.

21 Litwińska Z, Machaliński B. MiRNAs in chronic myeloid leukemia: small molecules, essential function. Leuk Lymphoma. 2017; 58(6):1297-305.

22 Kabekkodu SP, Shukla V, Varghese VK, Adiga D, Jishnu PV, Chakrabarty S, et al. Cluster miRNAs and cancer: diagnostic, prognostic and therapeutic opportunities. Wiley Interdiscip Rev RNA. 2020;11(2):e1563.

23 Tai-You H, MicroRNAs in human diseases: from cancer to cardiovascular disease. Immune Netw. 2011;11(3):135-54.

24 Wang IK, Li C-Y. Mir-20a-5p mediates hypoxia-induced autophagy by targeting ATG16L1 in acute kidney injury. Crit Care. 2014;18:P15.

25 Zhou Y, Xiong M, Fang L, Jiang L, Wen P, Dai $\mathrm{C}$, et al. miR-21-containing microvesicles from injured tubular epithelial cells promote tubular phenotype transition by targeting PTEN protein. Am J Pathol. 2013;183(4):1183-96.

26 Yao J, Xu C, Fang Z, Li Y, Liu H, Wang Y, et al. Androgen receptor regulated microrna miR-182-5p promotes prostate cancer progression by targeting the ARRDC3/ITGB4 pathway. Biochem Biophys Res Commun. 2016;474(1):213-9.

27 Li H, Ma Y, Chen B, Shi J. miR-182 enhances acute kidney injury by promoting apoptosis involving the targeting and regulation of TCF7L2/Wnt/ $\beta$-catenins pathway. Eur J Pharmacol. 2018;831:20-7.

28 Apostol AC, Beaudin AE. Reversing time: EZH1 deficiency hastens definitive hematopoiesis. Cell Stem Cell. 2018;22(3):285-7.

29 Zhou W, Wang S, Yang L, Sun Y, Zhang Q, Li $\mathrm{B}$, et al. Reference genes for qRT-PCR nor- malisation in different tissues, developmental stages, and stress conditions of hypericum perforatum. PeerJ. 2019;7:e7133.

30 Jackson JB, Hanson MR, Johnson GM, Spahlinger TG, Polesky HF, Bowman RJ. Longterm follow-up of blood donors with indeterminate human immunodeficiency virus type 1 results on Western blot. Transfusion. 2003; 35(2):98-102.

31 Sethi J, Kumar V, Rathi M, Gupta KL. Acute kidney injury associated with hypercalcaemic crisis in a patient with primary hyperparathyroidism. BMJ Case Rep. 2018;2018: bcr2018225235.

32 Verma S, Agarwal S, Singh S, Pandey N, Gupta S. Letter to editor: terlipressin is superior to noradrenaline in the management of acute kidney injury in acute on chronic liver failure. Hepatology. 2018;68(6):2442.

33 Serinan E, Altun Z, Aktaş S, Çeçen E, Olgun N. Comparison of cisplatin with lipoplatin in terms of ototoxicity. J Int Adv Otol. 2018; 14(2):211.

34 Jiang K, Zhang C, Yu B, Chen B, Liu Z, Hou $\mathrm{C}$, et al. Autophagic degradation of FOXO3a represses the expression of puma to block cell apoptosis in cisplatin-resistant osteosarcoma cells. Am J Cancer Res. 2017;7(7):1407-22.

35 Simion V, Haemmig S, Feinberg MW. Lncrnas in vascular biology and disease. Vascul Pharmacol. 2019;114:145.

36 Yu D, Tang C, Liu P, Qian W, Sheng L. Targeting lncrnas for cardiovascular therapeutics in coronary artery disease. Current Pharmaceutical Design. 2019;24.

37 Zuo N, Li Y, Liu N, Wang L. Differentially expressed long non-coding RNAs and mRNAs in patients with IgA nephropathy. Mol Med Rep. 2017;16(5):7724.

38 Li L, Sun R, Liang Y, Pan X, Li Z, Bai P, et al. Association between polymorphisms in long non-coding RNA PRNCR1 in 8q24 and risk of colorectal cancer. J Exp Clin Cancer Res. 2013;32(1): 104

39 Yan J, Bu X, Li Z, Wu J, Wang J. Screening the expression of several miRNAs from TaqMan low density array in traumatic brain injury: miR9a regulates neuronal apoptosis by modulating CCNA 2 and CACUL 1. J Neurochem. 2019;150(2):202-17.

40 Ashraf NM, Imran K, Kastner DW, Ikram K Mushtaq A, Hussain A, et al. Potential involvement of mi-RNA 574-3p in progression of prostate cancer: a bioinformatic study. $\mathrm{Mol}$ Cell Probes. 2017;36:21.

41 Che MW, Yan J, Gong LF, Geng XP, Li-Jie HE, Li-Zhong JU, et al. The role of SRF-miRNA-143-KLF-4 signal pathway in the HPMC phenotypic transition induced via high glucose. Med J Chin Peoples Liberation Army. 2016.

42 Lorenzen JM, Kielstein JT, Hafer C, Gupta SK, Kümpers P, Faulhaber-Walter R, et al. Circulating miR-210 predicts survival in critically ill patients with acute kidney injury. Clin J Am Soc Nephrol. 2011;6(7):15406.

43 Jiang Y, Zhou Z, Fei R, Zhou X, Chen T. Role of miR-182-5p overexpression in trichloroethylene-induced abnormal cell cycle functions in human HEPG2 cells. J Toxicol Environ Health A. 2019;82:1-8.

44 Li Q, Shen W, Li X, Zhang L, Jin X. The lncRNA n340790 accelerates carcinogenesis of thyroid cancer by regulating miR-1254. Am J Transl Res. 2017;9(5):2181-94.

45 Hidalgo I, Herrera-Merchan A, Ligos JM, Carramolino L, Nuñez J, Martinez F, et al. EZH1 is required for hematopoietic stem cell maintenance and prevents senescence-like cell cycle arrest. Cell Stem Cell. 2012;11(5): 649-62. 